NISSUNA UMANA INVESTIGAZIONE SI PUO DIMANDARE VERA SCIENZIA S'ESSA NON PASSA PER LE MATEMATICHE DIMOSTRAZIONI LEONARDO DA VINCI

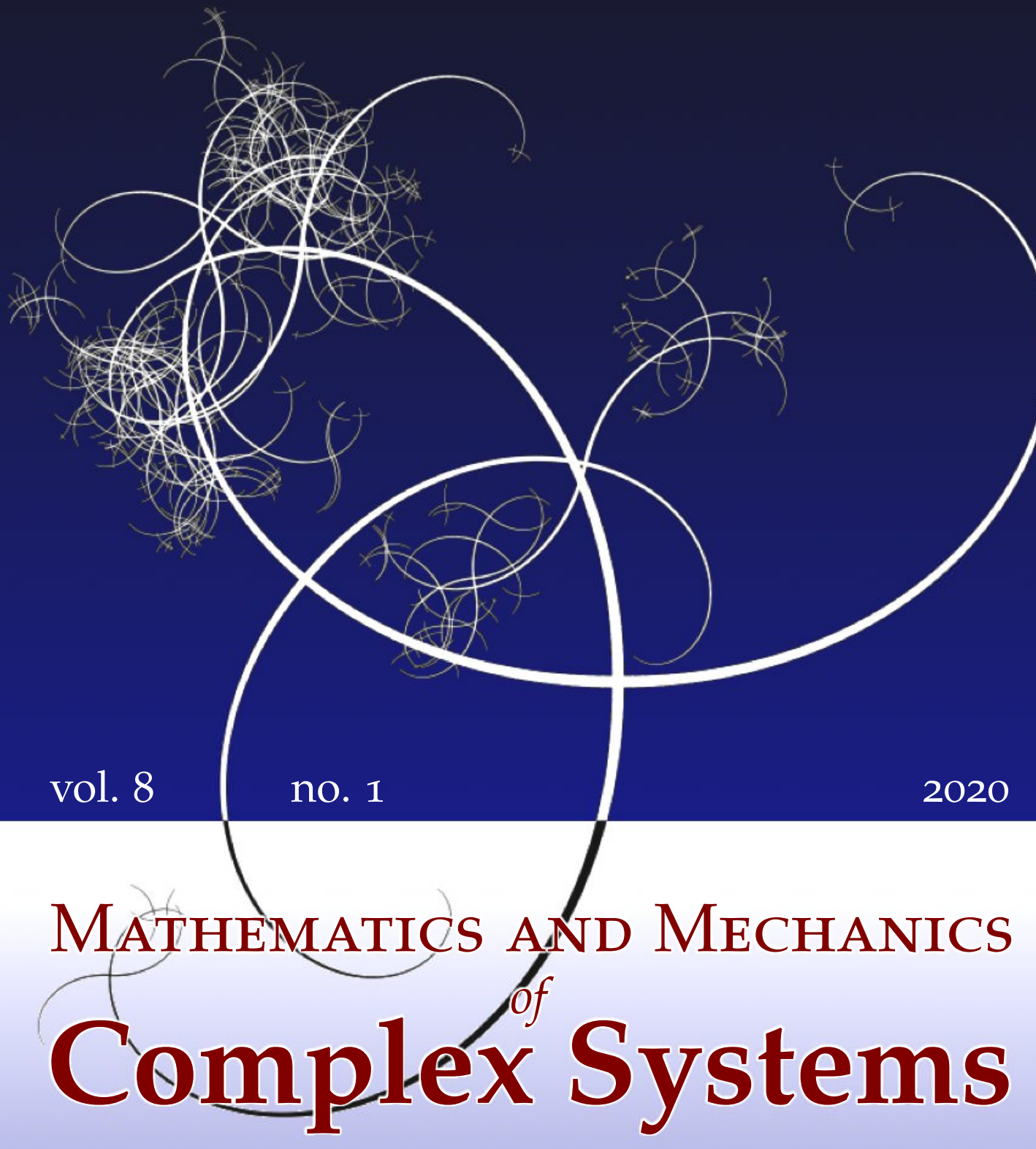

FABRIZIO DAVÌ

ON THE GENERALIZATION OF THE BREWSTER LAW 


\title{
ON THE GENERALIZATION OF THE BREWSTER LAW
}

\author{
FABRIZIO DAVÌ
}

\begin{abstract}
In classical photoelasticity of transparent isotropic materials, the Brewster law states that the difference in principal refraction index (birefringence) is proportional to the difference in principal stress. Here we show that such a relation can be generalized to anisotropic crystals only for the high-symmetry classes of the cubic group and for a specific plane stress, also for the high-symmetry classes of the trigonal, tetragonal, and hexagonal groups. No further generalizations are possible.
\end{abstract}

\section{Introduction}

One of the most important relations in photoelasticity is the one which, for transparent isotropic materials, shows that the difference in the principal refraction index (birefringence) is proportional to the difference in the principal stress [Aben and Guillemet 1993]:

$$
n_{i}-n_{j}=f_{\text {iso }}\left(\sigma_{i}-\sigma_{j}\right), \quad i, j=1,2,3, i \neq j,
$$

where $n_{i}$ are the principal refraction indexes, $\sigma_{i}$ are the principal values of the stress tensor $\boldsymbol{T}$, and $f_{\text {iso }}$ is the photoelastic constant for isotropic materials which depends on the components of the piezooptic tensor and on the refraction index $n_{o}$ of the unstressed material

Such a relation, obtained for the first time by David Brewster [1830] in 1818 and accordingly known as the "Brewster law", is at the basis of the experimental stress analysis based on photoelasticity. Besides the classical field of application in experimental structural mechanics (viz., [Kuske and Robertson 1974; Bain 2019]) such a relation allows, for instance, for the characterization and the quality control of high-energy physics crystals like the ones used in the CMS calorimeter at CERN or in the PANDA experiment at GSI in Darmstadt (viz., the recent review in [Montalto et al. 2019]).

\section{Communicated by Francesco dell'Isola.}

MSC2010: 78A10, 74F15, 74E10.

Keywords: photoelasticity, photoelastic constant, anisotropic crystals, Brewster law, scintillating crystals. 
When we look at (1) from a mechanical point of view, by keeping in mind the much celebrated Mohr's circle [Sokolnikoff 1956], we see that it states the direct proportionality between the birefringence in the plane orthogonal to the $k$-th principal direction of stress and the shear stress in the same plane:

$$
(\Delta n)_{k}=2 f_{\text {iso }} \tau_{k}, \quad k=1,2,3,
$$

where

$$
(\Delta n)_{k}=n_{i}-n_{j}, \quad \tau_{k}=\frac{\sigma_{i}-\sigma_{j}}{2}, \quad i, j=1,2,3, i \neq j \neq k .
$$

The experimental usefulness of such a relation thus becomes clear: the maximum birefringence is proportional to the maximum shear and accordingly can be related to failure criteria. On the other hand, provided we known the principal stress, a measurement of birefringence may allow for an experimental measurement of some components of the piezooptic tensor.

Here we look at the possibility to generalize the Brewster law for anisotropic materials to a more general relation in which the birefringences $(\Delta n)_{k}, k=1,2,3$, are linear combinations of the three shear stresses $\tau_{j}, j=1,2,3$, with the coefficients $f_{k j}$ of the linear combinations depending on the refraction index of the unstressed material, on the components of the piezooptic tensor, and on the angle between the principal optical and stress directions: ${ }^{1}$

$$
(\Delta n)_{k}=(\Delta n)_{k}^{o}+f_{k j} \tau_{j}, \quad k, j=1,2,3 .
$$

We look in detail at optically isotropic, uniaxial, and biaxial crystals and we show that, in order to arrive at a relation like (4), the birefringence must not depend on the spherical stress, an instance which depends on the crystal symmetry group (besides the trivial case of purely deviatoric (traceless) stresses). We show that this is possible for any stress in optically isotropic crystals, besides the isotropic case, only for the high-symmetry classes of the cubic group; moreover, when the stress has only diagonal component the obtained relation (4) simplifies into the isotropic one.

When we deal with optically uniaxial and biaxial crystals we show that in the general case it is not possible to arrive at (4). However, for uniaxial crystal we show that, for plane stress in the plane orthogonal to the material symmetry axis and provided the stressed crystal remains uniaxial, we can arrive at the isotropic relation (1) in the same plane for the high-symmetry trigonal, tetragonal, and hexagonal crystal classes.

${ }^{1}$ The same issue was addressed in [Rinaldi et al. 2018]; however, the results obtained there were related to specific cases of stress without the formal and more general treatment we give here; further, the treatment of cubic crystal was missing. 
No other generalizations of the Brewster law are possible, besides for very specific material and particular cases of stress.

\section{Crystal photoelasticity}

2.1. Propagation of light in crystals. We consider a three-dimensional region composed of a polarized, nonconducting, and nonmagnetizable material: then for $\boldsymbol{e}$ and $\boldsymbol{h}$ the electric and magnetic fields, respectively, a monochromatic and linearly polarized light is the electromagnetic plane wave [Born and Wolf 1999]:

$$
\boldsymbol{e}=\boldsymbol{e}_{0} \cos \omega\left(t-\frac{\boldsymbol{m} \cdot \boldsymbol{x}}{v}\right), \quad \boldsymbol{h}=\boldsymbol{h}_{0} \cos \omega\left(t-\frac{\boldsymbol{m} \cdot \boldsymbol{x}}{v}\right), \quad v=\frac{c}{n},
$$

where $\boldsymbol{e}_{0}$ and $\boldsymbol{h}_{0}$ are the constant amplitudes, $\omega$ is the frequency, $\boldsymbol{m}$ is the direction of propagation, $\|\boldsymbol{m}\|=1, v$ is the velocity of propagation of electromagnetic fields in a medium, $c$ is the light velocity in the vacuum, and $|n|<1$ is the refraction index.

The propagation condition for (5) is provided by the Maxwell equations with null total charge density,

$$
\operatorname{curl} \boldsymbol{e}=-\mu_{o} \frac{\partial \boldsymbol{h}}{\partial t}, \quad \operatorname{div} \boldsymbol{d}=0, \quad \operatorname{curl} \boldsymbol{h}=\frac{\partial \boldsymbol{d}}{\partial t}, \quad \operatorname{div} \boldsymbol{h}=0,
$$

where $\boldsymbol{d}$ denotes the electric displacement and $\mu_{0}$ is the vacuum permeability.

For an anisotropic dielectric material the constitutive relation between the electric field and the electric displacement is given by [Sirotin and Shaskolskaya 1982; Perelomova and Tagieva 1983; Nye 1985] ${ }^{2}$

$$
\boldsymbol{e}=\epsilon_{0}^{-1} \boldsymbol{B} \boldsymbol{d},
$$

where $\boldsymbol{B} \in \mathrm{Sym}^{+}$is the dielectric rigidity (or dielectric impermeability or inverse permittivity) tensor, normalized with respect to the dielectric permittivity of the vacuum $\epsilon_{0}$.

By using (5) and (7) in (6) and since $c^{2}=\epsilon_{o} \mu_{o}$, then we arrive at the propagation condition

$$
\left(\boldsymbol{M}(\boldsymbol{m})-n^{-2} \boldsymbol{P}^{\perp}(\boldsymbol{m})\right) \boldsymbol{d}=\mathbf{0},
$$

where the characteristic tensor $\boldsymbol{M}(\boldsymbol{m}) \in$ Sym is defined by

$$
\boldsymbol{M}(\boldsymbol{m})=\boldsymbol{P}^{\perp}(\boldsymbol{m}) \boldsymbol{B} \boldsymbol{P}^{\perp}(\boldsymbol{m}), \quad \boldsymbol{P}^{\perp}(\boldsymbol{m})=\boldsymbol{I}-\boldsymbol{m} \otimes \boldsymbol{m} ;
$$

here $\boldsymbol{P}^{\perp}(\boldsymbol{m})$ is the orthogonal projection on the plane normal to $\boldsymbol{m}$ and accordingly $\boldsymbol{M}(\boldsymbol{m})$ is the restriction of $\boldsymbol{B}$ to the plane orthogonal to $\boldsymbol{m}$.

\footnotetext{
${ }^{2}$ In the sequel we shall denote with Sym the subspace of symmetric tensors and $\mathrm{Sym}^{+}$the subspace of positive-definite symmetric tensors; Dev is the subspace of traceless symmetric tensors whereas Orth $^{+}$denotes the proper orthogonal group.
} 


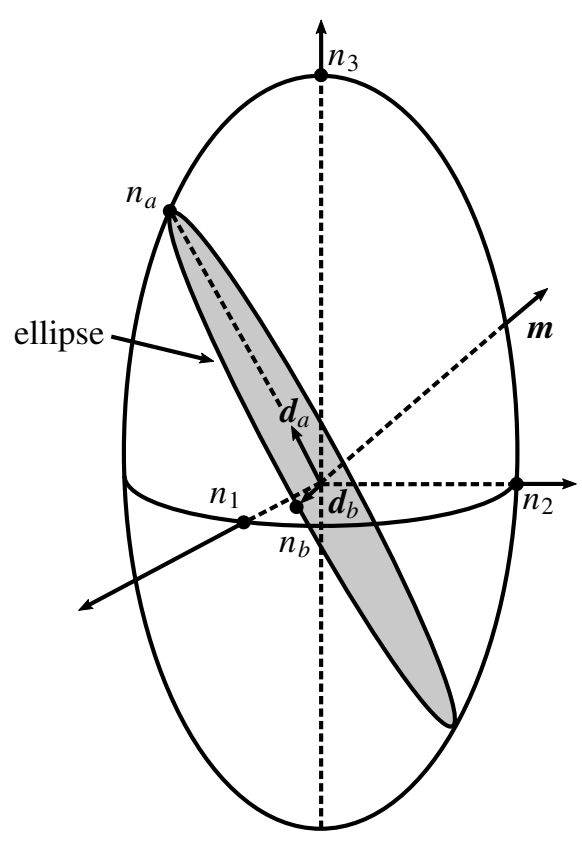

Figure 1. The optical indicatrix.

By the positive-definiteness of $\boldsymbol{B}$ we can define the optical indicatrix or conjugate index ellipsoid that is the locus of normalized constant dielectric energy [Born and Wolf 1999], which summarizes at a glance all the information about the crystal optical anisotropy:

$$
\boldsymbol{B} z \cdot \boldsymbol{z}=1,
$$

where $\boldsymbol{z}$ is a normalized electric displacement; hence $\boldsymbol{M}(\boldsymbol{m})$ is associated with the ellipse we get by intersecting the optical indicatrix with the plane orthogonal to $\boldsymbol{m}$ through the ellipsoid center. See Figure 1.

The eigenvalue problem associated with the propagation condition (8) admits, for each direction of propagation $\boldsymbol{m}$, at most two eigencouples

$$
\left(n_{a}^{-2}, \boldsymbol{d}_{a}\right), \quad\left(n_{b}^{-2}, \boldsymbol{d}_{b}\right)
$$

whose eigenvectors are mutually orthogonal and lie in the plane orthogonal to $\boldsymbol{m}$ : clearly the eigenvalues are the semiaxis of the ellipse described by $\boldsymbol{M}(\boldsymbol{m})$ and whose directions are spanned by the eigenvectors.

Whenever $n_{a} \neq n_{b}$ we have two different velocities $v_{a} \neq v_{b}$ associated with a given direction of propagation $\boldsymbol{m}$ : such a phenomena is called double refraction and is measured in terms of the birefringence $\Delta n$ :

$$
\Delta n=n_{a}-n_{b}
$$


it makes sense to search for the directions of propagation $\boldsymbol{m}$ such that $\Delta n=0$ and no double refraction holds. We define optic axis as these propagation directions and there are at most three possibilities, depending on the multiplicity of the eigenvalues of $\boldsymbol{B}$.

- Three equal eigenvalues:

$$
\boldsymbol{B}=n_{o}^{-2} \boldsymbol{I} .
$$

The material is optically isotropic, each direction is an optic axis, and there is no birefringence.

- Two equal eigenvalues:

$$
\boldsymbol{B}=n_{o}^{-2}(\boldsymbol{I}-\boldsymbol{e} \otimes \boldsymbol{e})+n_{e}^{-2} \boldsymbol{e} \otimes \boldsymbol{e}, \quad\|\boldsymbol{e}\|=1 .
$$

The material is optically uniaxial, the direction $\boldsymbol{e}$ is the unique optic axis, and the maximum birefringence is attained for propagation directions orthogonal to $\boldsymbol{e}$ :

$$
(\Delta n)_{\max }=n_{e}-n_{o} .
$$

We say $n_{e}$ and $n_{o}$ are the extraordinary and ordinary refraction indices and we define a crystal to be optically positive or negative when $n_{e}<n_{o}$ or $n_{e}>n_{o}$, respectively.

- Three different eigenvalues $B_{1}>B_{2}>B_{3}$ :

$$
\boldsymbol{B}=B_{1} \boldsymbol{e}_{1} \otimes \boldsymbol{e}_{1}+B_{2} \boldsymbol{e}_{2} \otimes \boldsymbol{e}_{2}+B_{3} \boldsymbol{e}_{3} \otimes \boldsymbol{e}_{3}, \quad B_{i}=n_{i}^{-2} .
$$

The material is called optically biaxial; whenever the propagation direction coincides with an optic axis the ellipse associated with $\boldsymbol{M ( m )}$ degenerates into a circle which lies in the plane containing $\boldsymbol{e}_{2}$; then there are two optic axes orthogonal to $\boldsymbol{e}_{2}$ and bisected by the direction spanned by $\boldsymbol{e}_{1}$.

We have three birefringences corresponding to directions of propagation $\boldsymbol{m}=\boldsymbol{u}_{3}$, $\boldsymbol{m}=\boldsymbol{u}_{2}$, and $\boldsymbol{m}=\boldsymbol{u}_{1}$, respectively:

$$
(\Delta n)_{3}=n_{1}-n_{2}, \quad(\Delta n)_{2}=n_{3}-n_{1}, \quad(\Delta n)_{1}=n_{2}-n_{3},
$$

with

$$
(\Delta n)_{\max }=\sup \left\{\left|(\Delta n)_{1}\right|,\left|(\Delta n)_{2}\right|,\left|(\Delta n)_{3}\right|\right\} .
$$

In biaxial crystals, if the value of intermediate refractive index is closer to that of highest refractive index, the crystal is optically negative, and if it is closer to lowest refractive index, then the crystal is optically positive.

2.2. Photoelasticity of crystals. In photoelastic crystals the dielectric impermeability is a linear function of the Cauchy stress tensor $\boldsymbol{T} \in \mathrm{Sym}$ :

$$
\boldsymbol{B}(\boldsymbol{T})=\boldsymbol{B}_{o}+\mathbb{M}[\boldsymbol{T}],
$$


where $\mathbb{M}:$ Sym $\rightarrow$ Sym is the fourth-order Maxwell piezooptic tensor and $\boldsymbol{B}_{o}$ is the dielectric permeability in the unstressed state. We define the symmetry group $\mathcal{G}$ for a photoelastic crystal as

$\mathscr{G} \equiv\left\{\boldsymbol{Q} \in \mathrm{Orth}^{+} \mid \boldsymbol{Q M}[\boldsymbol{T}] \boldsymbol{Q}^{T}=\mathbb{M}\left[\boldsymbol{Q T} \boldsymbol{Q}^{T}\right]\right.$ and $\boldsymbol{Q} \boldsymbol{B}_{o} \boldsymbol{Q}^{T}=\boldsymbol{B}_{o}$ for all $\left.\boldsymbol{T} \in \mathrm{Sym}\right\}$.

Let $\Sigma \equiv\left\{\boldsymbol{e}_{1}, \boldsymbol{e}_{2}, \boldsymbol{e}_{3}\right\}$ be an orthonormal frame; then the components of the piezooptic tensor obeys

$$
\mathbb{M}_{i j h k}=\mathbb{M}_{j i h k}=\mathbb{M}_{i j k h}, \quad i, j, h, k=1,2,3,
$$

and the tabular form of these components for the various crystallographic classes and groups is provided in the Appendix.

As far the tensor $\boldsymbol{B}_{o}$ is concerned, in the frame $\Sigma$ its matrix has six independent components for crystals of the triclinic group, whereas for the crystals of the monoclinic group (all classes) we have

$$
\boldsymbol{B}_{o} \equiv\left[\begin{array}{ccc}
B_{11} & B_{12} & 0 \\
\cdot & B_{22} & 0 \\
\cdot & \cdot & B_{33}
\end{array}\right],
$$

where $\boldsymbol{e}_{3}$ is directed as the monoclinic $b$-axis. For orthorhombic crystals (all groups) we have instead

$$
\boldsymbol{B}_{o} \equiv\left[\begin{array}{ccc}
B_{11} & 0 & 0 \\
\cdot & B_{22} & 0 \\
\cdot & \cdot & B_{33}
\end{array}\right]
$$

in all the three cases $\boldsymbol{B}$ admits the representation (16) and hence triclinic, monoclinic, and orthorhombic crystals are optically biaxial.

Crystals of the tetragonal, trigonal, and hexagonal group (all classes) are optically uniaxial, since $\boldsymbol{B}_{o}$ admits the representation (14):

$$
\boldsymbol{B}_{o} \equiv\left[\begin{array}{ccc}
B_{11} & 0 & 0 \\
\cdot & B_{11} & 0 \\
\cdot & \cdot & B_{33}
\end{array}\right],
$$

where $\boldsymbol{e}_{3}$ is directed in this case as the symmetry $c$-axis.

The tensor $\boldsymbol{B}_{o}$ for cubic crystals and isotropic materials has the representation (13) and the material is optically isotropic.

The stress $\boldsymbol{T}$ changes the optical properties of materials: indeed an isotropic material can become uniaxial or biaxial when stressed, and a uniaxial one can behave biaxially upon the application of a stress: for a complete description of such changes see the analysis presented in [Davì 2015]. 


\section{The generalization of the Brewster law for anisotropic crystals}

For optically isotropic materials we have the following the stress-optic relation called the Brewster law (viz., [Aben and Guillemet 1993]):

$$
n_{i}-n_{k}=f_{\text {iso }}\left(\sigma_{i}-\sigma_{k}\right), \quad i, k=1,2,3, i \neq k, \quad f_{\text {iso }}=n_{o}^{3} \frac{\mathbb{M}_{1122}-\mathbb{M}_{1111}}{2},
$$

where $n_{o}$ is the isotropic refraction index and $\left(\sigma_{1}, \sigma_{2}, \sigma_{3}\right)$ are the principal components of $\boldsymbol{T}$. From a mechanical point of view (24) relates the birefringences (17) with (twice) the shear stress in the same plane

Here we wish to obtain the restrictions on the piezooptic tensor and on the stress which allow us to generalize (24) for anisotropic crystals to a relation between the birefringences (17) and all (twice) the shear stresses:

$$
(\Delta n)_{k}=(\Delta n)_{k}^{o}+f_{1 k}\left(\sigma_{2}-\sigma_{3}\right)+f_{2 k}\left(\sigma_{3}-\sigma_{1}\right)+f_{3 k}\left(\sigma_{1}-\sigma_{2}\right), \quad k=1,2,3,
$$

with $(\Delta n)_{k}^{o}$ depending on $\boldsymbol{B}_{o}$ and the constant $f_{i k}$ depending on both $\boldsymbol{B}_{o}$ and $\mathbb{M}$.

Let $\left(\sigma_{k}, \boldsymbol{w}_{k}\right)$ be the eigencouples of a generic stress $\boldsymbol{T}$ with

$$
\boldsymbol{w}_{k}=\boldsymbol{R} \boldsymbol{e}_{k}, \quad k=1,2,3, \boldsymbol{R} \in \mathrm{Orth}^{+} ;
$$

then by the decomposition of $\boldsymbol{T}$ into its spherical and deviatoric parts

$$
\boldsymbol{T}=\sigma_{m} \boldsymbol{I}+\operatorname{dev} \boldsymbol{T}, \quad \sigma_{m}=\frac{1}{3}\left(\sigma_{1}+\sigma_{2}+\sigma_{3}\right),
$$

we get, in the frame $\Sigma_{\boldsymbol{T}} \equiv\left\{\boldsymbol{w}_{1}, \boldsymbol{w}_{2}, \boldsymbol{w}_{3}\right\}$,

$$
\operatorname{dev} \boldsymbol{T} \equiv \frac{1}{3}\left[\begin{array}{ccc}
2 \sigma_{1}-\sigma_{2}-\sigma_{3} & 0 & 0 \\
\cdot & 2 \sigma_{2}-\sigma_{1}-\sigma_{3} & 0 \\
\cdot & \cdot & 2 \sigma_{3}-\sigma_{1}-\sigma_{2}
\end{array}\right],
$$

which can be rewritten in terms of the shear stresses

as

$$
\tau_{1}=\frac{\sigma_{2}-\sigma_{3}}{2}, \quad \tau_{2}=\frac{\sigma_{3}-\sigma_{1}}{2}, \quad \tau_{3}=\frac{\sigma_{1}-\sigma_{2}}{2}
$$

$$
\operatorname{dev} \boldsymbol{T} \equiv \frac{2}{3}\left[\begin{array}{ccc}
\tau_{3}-\tau_{2} & 0 & 0 \\
\cdot & \tau_{1}-\tau_{3} & 0 \\
\cdot & \cdot & \tau_{2}-\tau_{1}
\end{array}\right]
$$

By (19), (27), and (30) then

$$
\begin{aligned}
\boldsymbol{B}\left(\sigma_{m}, \tau_{1}, \tau_{2}, \tau_{3}\right)= & \boldsymbol{B}_{o}+\sigma_{m} \mathbb{M}[\boldsymbol{I}]+\frac{2}{3}\left(\tau_{3}-\tau_{2}\right) \mathbb{M}\left[\boldsymbol{w}_{1} \otimes \boldsymbol{w}_{1}\right] \\
& +\frac{2}{3}\left(\tau_{1}-\tau_{3}\right) \mathbb{M}\left[\boldsymbol{w}_{2} \otimes \boldsymbol{w}_{2}\right]+\frac{2}{3}\left(\tau_{2}-\tau_{1}\right) \mathbb{M}\left[\boldsymbol{w}_{3} \otimes \boldsymbol{w}_{3}\right] \\
= & \boldsymbol{B}_{o}+\sigma_{m} \mathbb{M}[\boldsymbol{I}]+\frac{2}{3} \tau_{3} \mathbb{M}\left[\boldsymbol{w}_{1} \otimes \boldsymbol{w}_{1}-\boldsymbol{w}_{2} \otimes \boldsymbol{w}_{2}\right] \\
& +\frac{2}{3} \tau_{2} \mathbb{M}\left[\boldsymbol{w}_{3} \otimes \boldsymbol{w}_{3}-\boldsymbol{w}_{1} \otimes \boldsymbol{w}_{1}\right]+\frac{2}{3} \tau_{1} \mathbb{M}\left[\boldsymbol{w}_{2} \otimes \boldsymbol{w}_{2}-\boldsymbol{w}_{3} \otimes \boldsymbol{w}_{3}\right]
\end{aligned}
$$


Let $\left(B_{k}, \boldsymbol{u}_{k}\right)$ the eigencouples of $\boldsymbol{B}(\boldsymbol{T})$ with

$$
\boldsymbol{u}_{k}=\widehat{\boldsymbol{R}} \boldsymbol{e}_{k}, \quad k=1,2,3, \widehat{\boldsymbol{R}} \in \mathrm{Orth}^{+}
$$

then we have

$$
B_{k}\left(\sigma_{m}, \tau_{1}, \tau_{2}, \tau_{3}\right)=\boldsymbol{B}\left(\sigma_{m}, \tau_{1}, \tau_{2}, \tau_{3}\right) \cdot \boldsymbol{u}_{k} \otimes \boldsymbol{u}_{k}, \quad k=1,2,3, \text { not summed, }
$$

and by $(16)_{2}$ the principal refraction index of the stressed material is given by

$$
n_{k}\left(\sigma_{m}, \tau_{1}, \tau_{2}, \tau_{3}\right)=\frac{1}{\sqrt{B_{k}\left(\sigma_{m}, \tau_{1}, \tau_{2}, \tau_{3}\right)}},
$$

a relation that can be linearized into $n_{k}\left(\sigma_{m}, \tau_{1}, \tau_{2}, \tau_{3}\right)$

$$
=\frac{1}{\sqrt{B_{k}(\mathbf{0})}}-\frac{1}{2 B_{k}^{3 / 2}(\mathbf{0})}\left(\left.\frac{\partial B_{k}}{\partial \sigma_{m}}\right|_{\mathbf{0}} \sigma_{m}+\left.\sum_{j=1}^{3} \frac{\partial B_{k}}{\partial \tau_{j}}\right|_{\mathbf{0}} \tau_{j}\right)+o\left(\|\boldsymbol{T}\|^{2}\right) .
$$

By (31) and (32) (in all of the following relation $k$ is fixed and not summed)

$$
\begin{aligned}
B_{k}(\mathbf{0}) & =\boldsymbol{B}_{o} \cdot \widehat{\boldsymbol{R}} \boldsymbol{e}_{k} \otimes \widehat{\boldsymbol{R}} \boldsymbol{e}_{k}=B_{i j}^{o} \boldsymbol{e}_{i} \otimes \boldsymbol{e}_{j} \cdot \widehat{\boldsymbol{R}} \boldsymbol{e}_{k} \otimes \widehat{\boldsymbol{R}} \boldsymbol{e}_{k} \\
& =B_{i j}^{o}\left(\widehat{\boldsymbol{R}}_{k} \cdot \boldsymbol{e}_{i}\right)\left(\widehat{\boldsymbol{R}}_{k} \cdot \boldsymbol{e}_{j}\right)=B_{i j}^{o} \widehat{R}_{j k} \widehat{R}_{i k} ;
\end{aligned}
$$

moreover,

$$
\left.\frac{\partial B_{k}}{\partial \sigma_{m}}\right|_{\mathbf{0}}=\mathbb{M}[\boldsymbol{I}] \cdot \boldsymbol{u}_{k} \otimes \boldsymbol{u}_{k}=\mathbb{M}[\boldsymbol{I}] \cdot \widehat{\boldsymbol{R}} \boldsymbol{e}_{k} \otimes \widehat{\boldsymbol{R}} \boldsymbol{e}_{k}=\widehat{\boldsymbol{R}}^{T} \mathbb{M}[\boldsymbol{I}] \widehat{\boldsymbol{R}} \cdot \boldsymbol{e}_{k} \otimes \boldsymbol{e}_{k}=M_{k k},
$$

and by using also (26) finally we get

$$
\begin{aligned}
\left.\frac{\partial B_{k}}{\partial \tau_{1}}\right|_{\mathbf{0}} & =\frac{2}{3} \mathbb{M}\left[\boldsymbol{w}_{2} \otimes \boldsymbol{w}_{2}-\boldsymbol{w}_{3} \otimes \boldsymbol{w}_{3}\right] \cdot \boldsymbol{u}_{k} \otimes \boldsymbol{u}_{k} \\
& =\frac{2}{3} \widehat{\boldsymbol{R}}^{T} \mathbb{M}\left[\boldsymbol{R}\left(\boldsymbol{e}_{2} \otimes \boldsymbol{e}_{2}-\boldsymbol{e}_{3} \otimes \boldsymbol{e}_{3}\right) \boldsymbol{R}^{T}\right] \widehat{\boldsymbol{R}} \cdot \boldsymbol{e}_{k} \otimes \boldsymbol{e}_{k} \\
& =2\left(\overline{\mathbb{M}}_{22 k k}-\overline{\mathbb{M}}_{33 k k}\right), \\
\left.\frac{\partial B_{k}}{\partial \tau_{2}}\right|_{\mathbf{0}} & =\frac{2}{3} \mathbb{M}\left[\boldsymbol{w}_{3} \otimes \boldsymbol{w}_{3}-\boldsymbol{w}_{1} \otimes \boldsymbol{w}_{1}\right] \cdot \boldsymbol{u}_{k} \otimes \boldsymbol{u}_{k} \\
& =\frac{2}{3} \widehat{\boldsymbol{R}}^{T} \mathbb{M}\left[\boldsymbol{R}\left(\boldsymbol{e}_{3} \otimes \boldsymbol{e}_{3}-\boldsymbol{e}_{1} \otimes \boldsymbol{e}_{1}\right) \boldsymbol{R}^{T}\right] \widehat{\boldsymbol{R}} \cdot \boldsymbol{e}_{k} \otimes \boldsymbol{e}_{k} \\
& =2\left(\overline{\mathbb{M}}_{33 k k}-\overline{\mathbb{M}}_{11 k k}\right), \\
\left.\frac{\partial B_{k}}{\partial \tau_{3}}\right|_{\mathbf{0}} & =\frac{2}{3} \mathbb{M}\left[\boldsymbol{w}_{1} \otimes \boldsymbol{w}_{1}-\boldsymbol{w}_{1} \otimes \boldsymbol{w}_{1}\right] \cdot \boldsymbol{u}_{k} \otimes \boldsymbol{u}_{k} \\
& =\frac{2}{3} \widehat{\boldsymbol{R}}^{T} \mathbb{M}\left[\boldsymbol{R}\left(\boldsymbol{e}_{1} \otimes \boldsymbol{e}_{1}-\boldsymbol{e}_{2} \otimes \boldsymbol{e}_{2}\right) \boldsymbol{R}^{T}\right] \widehat{\boldsymbol{R}} \cdot \boldsymbol{e}_{k} \otimes \boldsymbol{e}_{k} \\
& =2\left(\overline{\mathbb{M}}_{11 k k}-\overline{\mathbb{M}}_{22 k k}\right),
\end{aligned}
$$


where

$$
\overline{\mathbb{M}}_{h h k k}=\frac{1}{3} \widehat{\boldsymbol{R}}^{T} \mathbb{M}\left[\boldsymbol{R}\left(\boldsymbol{e}_{h} \otimes \boldsymbol{e}_{h}\right) \boldsymbol{R}^{T}\right] \widehat{\boldsymbol{R}} \cdot \boldsymbol{e}_{k} \otimes \boldsymbol{e}_{k}, \quad h, k \text { fixed }
$$

By using (37), (38), and (29) in (35) we arrive at the linearized expression of the principal refraction index in terms of the differences between principal stress:

$$
\begin{aligned}
n_{1}\left(\sigma_{1}, \sigma_{2}, \sigma_{3}\right)= & \frac{1}{\sqrt{B_{1}(\mathbf{0})}}-\frac{1}{2 B_{1}^{3 / 2}(\mathbf{0})}\left(\sigma_{m} M_{11}+\left(\sigma_{2}-\sigma_{3}\right)\left(\overline{\mathbb{M}}_{2211}-\overline{\mathbb{M}}_{3311}\right)\right. \\
& \left.+\left(\sigma_{3}-\sigma_{1}\right)\left(\overline{\mathbb{M}}_{3311}-\overline{\mathbb{M}}_{1111}\right)+\left(\sigma_{1}-\sigma_{2}\right)\left(\overline{\mathbb{M}}_{1111}-\overline{\mathbb{M}}_{2211}\right)\right), \\
n_{2}\left(\sigma_{1}, \sigma_{2}, \sigma_{3}\right)= & \frac{1}{\sqrt{B_{2}(\mathbf{0})}}-\frac{1}{2 B_{2}^{3 / 2}(\mathbf{0})}\left(\sigma_{m} M_{22}+\left(\sigma_{2}-\sigma_{3}\right)\left(\overline{\mathbb{M}}_{2222}-\overline{\mathbb{M}}_{3322}\right)\right. \\
& \left.+\left(\sigma_{3}-\sigma_{1}\right)\left(\overline{\mathbb{M}}_{3322}-\overline{\mathbb{M}}_{1122}\right)+\left(\sigma_{1}-\sigma_{2}\right)\left(\overline{\mathbb{M}}_{1122}-\overline{\mathbb{M}}_{2222}\right)\right), \\
n_{3}\left(\sigma_{1}, \sigma_{2}, \sigma_{3}\right)= & \frac{1}{\sqrt{B_{3}(\mathbf{0})}}-\frac{1}{2 B_{3}^{3 / 2}(\mathbf{0})}\left(\sigma_{m} M_{33}+\left(\sigma_{2}-\sigma_{3}\right)\left(\overline{\mathbb{M}}_{2233}-\overline{\mathbb{M}}_{3333}\right)\right. \\
& \left.+\left(\sigma_{3}-\sigma_{1}\right)\left(\overline{\mathbb{M}}_{3333}-\overline{\mathbb{M}}_{1133}\right)+\left(\sigma_{1}-\sigma_{2}\right)\left(\overline{\mathbb{M}}_{1133}-\overline{\mathbb{M}}_{2233}\right)\right) .
\end{aligned}
$$

We are now in position to write the birefringences (17) as

$$
\begin{aligned}
& (\Delta n)_{1}=(\Delta n)_{1}^{o}+f_{1} \sigma_{m}+f_{11}\left(\sigma_{2}-\sigma_{3}\right)+f_{21}\left(\sigma_{3}-\sigma_{1}\right)+f_{31}\left(\sigma_{1}-\sigma_{2}\right), \\
& (\Delta n)_{2}=(\Delta n)_{2}^{o}+f_{2} \sigma_{m}+f_{12}\left(\sigma_{2}-\sigma_{3}\right)+f_{22}\left(\sigma_{3}-\sigma_{1}\right)+f_{32}\left(\sigma_{1}-\sigma_{2}\right), \\
& (\Delta n)_{3}=(\Delta n)_{3}^{o}+f_{3} \sigma_{m}+f_{13}\left(\sigma_{2}-\sigma_{3}\right)+f_{23}\left(\sigma_{3}-\sigma_{1}\right)+f_{33}\left(\sigma_{1}-\sigma_{2}\right),
\end{aligned}
$$

where

$$
\begin{aligned}
(\Delta n)_{1}^{o} & =B_{2}^{-1 / 2}(\mathbf{0})-B_{3}^{-1 / 2}(\mathbf{0}), \\
(\Delta n)_{2}^{o} & =B_{3}^{-1 / 2}(\mathbf{0})-B_{1}^{-1 / 2}(\mathbf{0}), \\
(\Delta n)_{3}^{o} & =B_{1}^{-1 / 2}(\mathbf{0})-B_{2}^{-1 / 2}(\mathbf{0}), \\
f_{1} & =\frac{1}{2 B_{3}^{3 / 2}(\mathbf{0})} M_{33}-\frac{1}{2 B_{2}^{3 / 2}(\mathbf{0})} M_{22}, \\
f_{2} & =\frac{1}{2 B_{1}^{3 / 2}(\mathbf{0})} M_{11}-\frac{1}{2 B_{3}^{3 / 2}(\mathbf{0})} M_{33}, \\
f_{3} & =\frac{1}{2 B_{2}^{3 / 2}(\mathbf{0})} M_{22}-\frac{1}{2 B_{1}^{3 / 2}(\mathbf{0})} M_{11},
\end{aligned}
$$


and with the components of the nonsymmetric matrix $\left[f_{i k}\right]$ given by

$$
\begin{aligned}
& f_{11}=\frac{1}{2 B_{2}^{3 / 2}(\mathbf{0})}\left(\overline{\mathbb{M}}_{3322}-\overline{\mathbb{M}}_{2222}\right)+\frac{1}{2 B_{3}^{3 / 2}(\mathbf{0})}\left(\overline{\mathbb{M}}_{2233}-\overline{\mathbb{M}}_{3333}\right), \\
& f_{12}=\frac{1}{2 B_{2}^{3 / 2}(\mathbf{0})}\left(\overline{\mathbb{M}}_{1122}-\overline{\mathbb{M}}_{3322}\right)+\frac{1}{2 B_{3}^{3 / 2}(\mathbf{0})}\left(\overline{\mathbb{M}}_{3333}-\overline{\mathbb{M}}_{1133}\right), \\
& f_{13}=\frac{1}{2 B_{2}^{3 / 2}(\mathbf{0})}\left(\overline{\mathbb{M}}_{2222}-\overline{\mathbb{M}}_{1122}\right)+\frac{1}{2 B_{3}^{3 / 2}(\mathbf{0})}\left(\overline{\mathbb{M}}_{1133}-\overline{\mathbb{M}}_{2233}\right), \\
& f_{21}=\frac{1}{2 B_{3}^{3 / 2}(\mathbf{0})}\left(\overline{\mathbb{M}}_{3333}-\overline{\mathbb{M}}_{2233}\right)+\frac{1}{2 B_{1}^{3 / 2}(\mathbf{0})}\left(\overline{\mathbb{M}}_{2211}-\overline{\mathbb{M}}_{3311}\right), \\
& f_{22}=\frac{1}{2 B_{3}^{3 / 2}(\mathbf{0})}\left(\overline{\mathbb{M}}_{1133}-\overline{\mathbb{M}}_{3333}\right)+\frac{1}{2 B_{1}^{3 / 2}(\mathbf{0})}\left(\overline{\mathbb{M}}_{3311}-\overline{\mathbb{M}}_{1111}\right), \\
& f_{23}=\frac{1}{2 B_{3}^{3 / 2}(\mathbf{0})}\left(\overline{\mathbb{M}}_{2233}-\overline{\mathbb{M}}_{1133}\right)+\frac{1}{2 B_{1}^{3 / 2}(\mathbf{0})}\left(\overline{\mathbb{M}}_{1111}-\overline{\mathbb{M}}_{2211}\right), \\
& f_{31}=\frac{1}{2 B_{1}^{3 / 2}(\mathbf{0})}\left(\overline{\mathbb{M}}_{3311}-\overline{\mathbb{M}}_{2211}\right)+\frac{1}{2 B_{2}^{3 / 2}(\mathbf{0})}\left(\overline{\mathbb{M}}_{2222}-\overline{\mathbb{M}}_{3322}\right), \\
& f_{32}=\frac{1}{2 B_{1}^{3 / 2}(\mathbf{0})}\left(\overline{\mathbb{M}}_{1111}-\overline{\mathbb{M}}_{3311}\right)+\frac{1}{2 B_{2}^{3 / 2}(\mathbf{0})}\left(\overline{\mathbb{M}}_{3322}-\overline{\mathbb{M}}_{1122}\right), \\
& f_{33}=\frac{1}{2 B_{1}^{3 / 2}(\mathbf{0})}\left(\overline{\mathbb{M}}_{2211}-\overline{\mathbb{M}}_{1111}\right)+\frac{1}{2 B_{2}^{3 / 2}(\mathbf{0})}\left(\overline{\mathbb{M}}_{1122}-\overline{\mathbb{M}}_{2222}\right)
\end{aligned}
$$

Relations (41) differ from the desired expression (25) by the presence of the terms in $\sigma_{m}$ : at a glance, we see that the necessary condition to have a generalized Brewster law (25) is that either $\boldsymbol{T}$ is traceless or, in the general case,

$$
f_{1}=f_{2}=f_{3}=0,
$$

a condition which, in terms of the piezooptic tensor $\mathbb{M}$ and the inverse permittivity $\boldsymbol{B}_{o}$, reads

$$
\begin{aligned}
& B_{2}^{2 / 3}(\mathbf{0}) M_{33}-B_{3}^{2 / 3}(\mathbf{0}) M_{22}=0, \\
& B_{3}^{2 / 3}(\mathbf{0}) M_{11}-B_{1}^{2 / 3}(\mathbf{0}) M_{33}=0, \\
& B_{1}^{2 / 3}(\mathbf{0}) M_{22}-B_{2}^{2 / 3}(\mathbf{0}) M_{11}=0 ;
\end{aligned}
$$

it is easy to show that (46) admits the solution

$$
M_{k k}=\alpha B_{k}^{2 / 3}(\mathbf{0}), \quad k=1,2,3, \alpha \in \mathbb{R} .
$$

In the following subsections we shall deal in detail with condition (47) and with the expression of the components of (25) for the various symmetry groups and classes: 
in particular we shall search for the cases in which (47) holds and those in which the matrix $\left[f_{i k}\right]$ is symmetric or diagonal.

3.1. Optically isotropic. For optically isotropic materials, the inverse permittivity has the representation (13): accordingly $B_{k}(\mathbf{0})=n_{o}^{-2}$ and $(\Delta n)_{k}^{o}=0$ for all $k$. As far as the piezooptic tensor and conditions (47) are concerned, we need to analyze in detail the various cases.

3.1.1. Isotropic materials. For isotropic materials the piezooptic tensor is symmetric and from (13), (19), and (78) of the Appendix we get

$$
\boldsymbol{B}(\boldsymbol{T})=n_{o}^{-2}+\sigma_{m}\left(\mathbb{M}_{1111}+2 \mathbb{M}_{1122}\right) \boldsymbol{I}+\left(\mathbb{M}_{1111}-\mathbb{M}_{1122}\right) \operatorname{dev} \boldsymbol{T},
$$

and the principal directions of $\boldsymbol{B}(\boldsymbol{T})$ coincide with those of $\operatorname{dev} \boldsymbol{T}$ with $\boldsymbol{R}=\widehat{\boldsymbol{R}}$; by the definition of isotropic group

$$
\widehat{\boldsymbol{R}}^{T} \mathbb{M}\left[\boldsymbol{R} \boldsymbol{A} \boldsymbol{R}^{T}\right] \widehat{\boldsymbol{R}}=\widehat{\boldsymbol{R}}^{T} \boldsymbol{R} \mathbb{M}[\boldsymbol{A}] \boldsymbol{R}^{T} \widehat{\boldsymbol{R}}=\mathbb{M}[\boldsymbol{A}] \text { for all } \boldsymbol{A} \in \mathrm{Sym},
$$

then we have

$$
\begin{aligned}
M_{k k} & =\mathbb{M}[\boldsymbol{I}] \cdot \boldsymbol{e}_{k} \otimes \boldsymbol{e}_{k}=\frac{1}{3}\left(\mathbb{M}_{1111}+2 \mathbb{M}_{1122}\right) \boldsymbol{I} \cdot \boldsymbol{e}_{k} \otimes \boldsymbol{e}_{k} \\
& =\frac{\mathbb{M}_{1111}+2 \mathbb{M}_{1122}}{3} \quad \text { for all } k,
\end{aligned}
$$

and condition (47) is verified for $3 \alpha=\left(\mathbb{M}_{1111}+2 \mathbb{M}_{1122}\right) n_{o}^{3}$.

Moreover, by (49), relation (39) yields

$$
\left.\overline{\mathbb{M}}_{h h k k}=\frac{1}{3} \mathbb{M}\left[\boldsymbol{e}_{h} \otimes \boldsymbol{e}_{h}\right)\right] \cdot \boldsymbol{e}_{k} \otimes \boldsymbol{e}_{k}=\frac{1}{3} \mathbb{M}_{k k h h}, \quad h, k \text { fixed, }
$$

and (44) gives in turn

$$
\begin{aligned}
f_{11}=f_{22}=f_{33} & =\frac{n_{o}^{3}}{2} \frac{2}{3}\left(\mathbb{M}_{1122}-\mathbb{M}_{1111}\right), \\
f_{12}=f_{12}=f_{13}=f_{31}=f_{23}=f_{32} & =-\frac{n_{o}^{3}}{2} \frac{1}{3}\left(\mathbb{M}_{1122}-\mathbb{M}_{1111}\right),
\end{aligned}
$$

and accordingly (41) reduces to (24).

3.1.2. Cubic crystals, classes $432, \overline{4} 3 m$, and $m 3 m$. Also in this case the piezooptic tensor is symmetric and from (76) of the Appendix we have

$$
\begin{aligned}
M_{k k} & =\widehat{\boldsymbol{R}}^{T} \mathbb{M}[\boldsymbol{I}] \widehat{\boldsymbol{R}} \cdot \boldsymbol{e}_{k} \otimes \boldsymbol{e}_{k}=\frac{1}{3}\left(\mathbb{M}_{1111}+2 \mathbb{M}_{1122}\right) \widehat{\boldsymbol{R}}^{T} \widehat{\boldsymbol{R}} \cdot \boldsymbol{e}_{k} \otimes \boldsymbol{e}_{k} \\
& =\frac{\mathbb{M}_{1111}+2 \mathbb{M}_{1122}}{3} \quad \text { for all } k ;
\end{aligned}
$$

then, even for these cubic classes condition (47) is verified for the same value of $\alpha$ as in the isotropic case and the Brewster law can be generalized to the form (25) with $(\Delta n)_{k}^{o}=0, k=1,2,3$. 
By (39) and (44) the components of $\left[f_{i k}\right]$ depend not only on the components of $\mathbb{M}$ but also on both $\boldsymbol{R}$ and $\widehat{\boldsymbol{R}}$ : however, if we assume that the frame $\Sigma$ is also the principal frame for $\boldsymbol{T}$, that is $\boldsymbol{R}=\boldsymbol{I}$, then by (76) we obtain again relation (48); accordingly the results for isotropic material still apply with the components of $\left[f_{i k}\right]$ given by (52) and (24), which holds even in this case.

3.1.3. Cubic crystals, classes 23 and $3 m$. The tensor $\mathbb{M}$ for these classes is not symmetric and hence we cannot apply the results of [Mehrabadi and Cowin 1990]. However, since

$$
\begin{aligned}
\mathbb{M}[\boldsymbol{I}]=\left(\mathbb{M}_{1111}+\mathbb{M}_{1122}+\mathbb{M}_{1133}\right) \boldsymbol{e}_{1} \otimes \boldsymbol{e}_{1} & +\left(\mathbb{M}_{1111}+\mathbb{M}_{1122}+\mathbb{M}_{2211}\right) \boldsymbol{e}_{2} \otimes \boldsymbol{e}_{2} \\
& +\left(\mathbb{M}_{1111}+\mathbb{M}_{2211}+\mathbb{M}_{3311}\right) \boldsymbol{e}_{3} \otimes \boldsymbol{e}_{3},
\end{aligned}
$$

then condition (47) cannot be verified for any $\widehat{\boldsymbol{R}}$ for a unique value of $\alpha$. Therefore for cubic crystal of these classes it is not possible, in general, to arrive at the Brewster-type relation (25).

3.2. Optically uniaxial. For optically uniaxial crystals, the inverse permittivity has the representation (14). We set $\boldsymbol{e}_{3}=\boldsymbol{e}$ and therefore,

$$
(\Delta n)_{1}^{o}=-(\Delta n)_{2}^{o}=n_{o}-n_{e}, \quad(\Delta n)_{3}^{o}=0,
$$

whereas condition (47) requires

$$
M_{11}=M_{22}=\alpha n_{o}^{2}, \quad M_{33}=\alpha n_{e}^{2} .
$$

Relation (56) may be verified for some specific crystals but, in the general case, cannot be satisfied and we may conclude that for uniaxial crystal the generalization (25) of the Brewster law is not possible, unless the stress is a purely deviatoric one.

Whereas it is still possible to arrive at (41), we may restrict our analysis to plane stress in the plane orthogonal to the optic axis, say $\boldsymbol{T} \boldsymbol{e}_{3}=\mathbf{0}$. In this case $\boldsymbol{e}_{3}=\boldsymbol{w}_{3}$ with $\sigma_{3}=0$ and $\boldsymbol{R}$ is a rotation about $\boldsymbol{e}_{3}$.

Further, if we choose $\alpha$ which satisfies (56), then we may write $(41)_{3}$ as

$$
n_{1}-n_{2}=f_{13} \sigma_{2}-f_{23} \sigma_{1}+f_{33}\left(\sigma_{1}-\sigma_{2}\right),
$$

which can be rewritten in the Brewster-like form

$$
n_{1}-n_{2}=\left(f_{33}-f_{13}\right)\left(\sigma_{1}-\sigma_{2}\right),
$$

provided the following condition holds:

$$
f_{13}=f_{23} .
$$

From (44), the requirement that (59) be verified for any refraction indices $n_{o}$ and $n_{e}$ is equivalent to the two conditions

$$
\overline{\mathrm{M}}_{1133}=\overline{\mathrm{M}}_{2233}, \quad \overline{\mathrm{M}}_{1111}+\overline{\mathrm{M}}_{1122}=\overline{\mathrm{M}}_{2222}+\overline{\mathrm{M}}_{2211} \text {. }
$$


Since $\boldsymbol{e}_{3}$ is an axis of symmetry for trigonal, tetragonal, and hexagonal crystals, the rotations about $\boldsymbol{e}_{3}$ belong to their symmetry group $\mathscr{G}$ and we can write (39) as $\overline{\mathbb{M}}_{h h k k}=\frac{1}{3} \boldsymbol{Q} \mathbb{M}\left[\boldsymbol{e}_{h} \otimes \boldsymbol{e}_{h}\right] \boldsymbol{Q}^{T} \cdot \boldsymbol{e}_{k} \otimes \boldsymbol{e}_{k}=\frac{1}{3} \mathbb{M}_{i j h h}\left(\boldsymbol{Q} \boldsymbol{e}_{i} \cdot \boldsymbol{e}_{k}\right)\left(\boldsymbol{Q} \boldsymbol{e}_{j} \cdot \boldsymbol{e}_{k}\right), \quad h, k$ fixed,

where $\boldsymbol{Q}=\widehat{\boldsymbol{R}}^{T} \boldsymbol{R} \in \mathrm{Orth}^{+}$.

Under the additional hypothesis that also $\widehat{\boldsymbol{R}}$ is a rotation about the material symmetry axis $\boldsymbol{e}_{3},{ }^{3}$ then we have that $\boldsymbol{Q} \boldsymbol{e}_{3}=\boldsymbol{e}_{3}$ and $\boldsymbol{Q} \boldsymbol{e}_{\alpha} \cdot \boldsymbol{e}_{3}=0, \alpha=1,2$. Therefore from (61) we can write

$$
\begin{aligned}
\overline{\mathbb{M}}_{1111}+\overline{\mathbb{M}}_{1122} & =\frac{1}{3}\left(\left(\mathbb{M}_{1111}+\mathbb{M}_{1122}\right) \cos ^{2} \varphi+\left(\mathbb{M}_{2222}+\mathbb{M}_{2211}\right) \sin ^{2} \varphi\right. \\
& \left.-\left(\mathbb{M}_{1211}+\mathbb{M}_{1222}+\mathbb{M}_{2111}+\mathbb{M}_{2122}\right) \sin \varphi \cos \varphi\right), \\
\overline{\mathbb{M}}_{2222}+\overline{\mathbb{M}}_{2211} & =\frac{1}{3}\left(\left(\mathbb{M}_{1111}+\mathbb{M}_{1122}\right) \sin ^{2} \varphi+\left(\mathbb{M}_{2222}+\mathbb{M}_{2211}\right) \cos ^{2} \varphi\right. \\
& \left.+\left(\mathbb{M}_{1211}+\mathbb{M}_{1222}+\mathbb{M}_{2111}+\mathbb{M}_{2122}\right) \sin \varphi \cos \varphi\right), \\
\overline{\mathbb{M}}_{1133} & =\frac{1}{3} \mathbb{M}_{3311}, \\
\overline{\mathbb{M}}_{2233} & =\frac{1}{3} \mathbb{M}_{3322},
\end{aligned}
$$

where $\varphi$ is the rotation angle about $\boldsymbol{e}_{3}$. Accordingly condition $(60)_{1}$ together with the requirement that $(60)_{2}$ holds for any value of $\varphi$ leads to the following restrictions on the symmetries of $\mathbb{M}$ :

$$
\begin{aligned}
\mathbb{M}_{3311} & =\mathbb{M}_{3322}, \\
\mathbb{M}_{1111}+\mathbb{M}_{1122} & =\mathbb{M}_{2222}+\mathbb{M}_{2211}, \\
\mathbb{M}_{1211}+\mathbb{M}_{1222}+\mathbb{M}_{2111}+\mathbb{M}_{2122} & =0
\end{aligned}
$$

From the tabular data in the Appendix it easy to show that these conditions hold only for the high-symmetry classes of trigonal $(\overline{3} \mathrm{~m}, 32,3 \mathrm{~m})$, tetragonal $(4 \mathrm{mmm}$, $422,4 / \mathrm{mm}$, and $\overline{4} 2 \mathrm{~m})$ and hexagonal $(\overline{6} \mathrm{~m} 2,622,6 \mathrm{~mm}$, and $6 / \mathrm{mmm})$ crystals, in which cases (58) reduces once again to (24).

3.3. Optically biaxial. In the case of optically biaxial crystals, the inverse permittivity has either the representation (21) or (22). In any case it is $(\Delta n)_{k}^{o} \neq 0$, $k=1,2,3$, and (47) leads to

$$
M_{11}=\alpha n_{1}^{2}, \quad M_{22}=\alpha n_{2}^{2}, \quad M_{33}=\alpha n_{2}^{2},
$$

and it is not possible to obtain the relation (25), unless (64) is verified for some specific crystals. However, it is possible to arrive at the relation (41) which on the other hand didn't simplify the expression for birefringence to an appreciable degree. Accordingly we didn't go into further details.

\footnotetext{
${ }^{3}$ This assumption means that the stressed crystal remains uniaxial: a complete characterization of the stresses which leave uniaxial crystal still uniaxial is provided in [Davì 2015].
} 


\section{Conclusions}

We looked at the possibility of generalizing the Brewster law for isotropic transparent materials to anisotropic crystalline materials. First we show that the birefringence can be written as a linear function of the spherical and shear stresses expressed as the difference in the principal stress, by taking into account the components of the unstressed inverse permittivity, those of the piezooptic tensor and the rotation of the stress, and the optical principal directions with respect to the symmetry axis of the material; then we show that this result can be formulated in a "Brewster-like" manner only when the birefringence is independent of the spherical part of the stress, a fact which is related to the crystal symmetry. We showed that the relation can be generalized for any stress only for the high-symmetry classes of the cubic group and that when the stress tensor is diagonal we obtain once more the isotropic relation.

For uniaxial crystals the relation can be generalized only for plane stress in the plane orthogonal to the optic axis and provided the stressed crystal remains uniaxial: also in this case we arrive at the isotropic-like relation in the plane of stress. We show that no general extension of the Brewster law is possible, in the general case, for biaxial crystals.

\section{Appendix}

In order to make the paper self-contained, in this appendix we list the tabular form of the piezooptic tensor $\mathbb{M}$ of the various crystallographic classes, from [Authier 2003].

Monoclinic. All classes:

$$
[\mathrm{M}] \equiv\left[\begin{array}{cccccc}
\mathbb{M}_{1111} & \mathbb{M}_{1122} & \mathbb{M}_{1133} & 0 & \mathbb{M}_{1113} & 0 \\
\mathbb{M}_{2211} & \mathbb{M}_{2222} & \mathbb{M}_{2233} & 0 & \mathbb{M}_{2213} & 0 \\
\mathbb{M}_{3311} & \mathbb{M}_{3322} & \mathbb{M}_{3333} & 0 & \mathbb{M}_{3313} & 0 \\
0 & 0 & 0 & \mathbb{M}_{2323} & 0 & \mathbb{M}_{2312} \\
\mathbb{M}_{1311} & \mathbb{M}_{1312} & \mathbb{M}_{1333} & 0 & \mathbb{M}_{1313} & 0 \\
0 & 0 & 0 & \mathbb{M}_{1223} & 0 & \mathbb{M}_{1212}
\end{array}\right] .
$$

Orthorhombic. All classes:

$$
[\mathbb{M}] \equiv\left[\begin{array}{cccccc}
\mathbb{M}_{1111} & \mathbb{M}_{1122} & \mathbb{M}_{1133} & 0 & 0 & 0 \\
\mathbb{M}_{2211} & \mathbb{M}_{2222} & \mathbb{M}_{2233} & 0 & 0 & 0 \\
\mathbb{M}_{3311} & \mathbb{M}_{3322} & \mathbb{M}_{3333} & 0 & 0 & 0 \\
0 & 0 & 0 & \mathbb{M}_{2323} & 0 & 0 \\
0 & 0 & 0 & 0 & \mathbb{M}_{1313} & 0 \\
0 & 0 & 0 & 0 & 0 & \mathbb{M}_{1212}
\end{array}\right] .
$$


Trigonal. Classes $\overline{3} m, 32$, and $3 m$ :

$$
[\mathbb{M}] \equiv\left[\begin{array}{cccccc}
\mathbb{M}_{1111} & \mathbb{M}_{1122} & \mathbb{M}_{1133} & \mathbb{M}_{1123} & 0 & 0 \\
\mathbb{M}_{1122} & \mathbb{M}_{1111} & \mathbb{M}_{1133} & -\mathbb{M}_{1123} & 0 & 0 \\
\mathbb{M}_{3311} & \mathbb{M}_{3311} & \mathbb{M}_{3333} & 0 & 0 & 0 \\
\mathbb{M}_{2311} & -\mathbb{M}_{2311} & 0 & \mathbb{M}_{2323} & 0 & 0 \\
0 & 0 & 0 & 0 & \mathbb{M}_{2323} & 2 \mathbb{M}_{2311} \\
0 & 0 & 0 & 0 & \mathbb{M}_{1123} & \mathbb{M}_{1111}-\mathbb{M}_{1122}
\end{array}\right] .
$$

Classes 3 and $\overline{3}$ :

$$
[\mathbb{M}] \equiv\left[\begin{array}{cccccc}
\mathbb{M}_{1111} & \mathbb{M}_{1122} & \mathbb{M}_{1133} & \mathbb{M}_{1123} & -\mathbb{M}_{2213} & 2 \mathbb{M}_{1222} \\
\mathbb{M}_{1122} & \mathbb{M}_{1111} & \mathbb{M}_{1133} & -\mathbb{M}_{1123} & \mathbb{M}_{2213} & -2 \mathbb{M}_{1222} \\
\mathbb{M}_{3311} & \mathbb{M}_{3311} & \mathbb{M}_{3333} & 0 & 0 & 0 \\
\mathbb{M}_{2311} & -\mathbb{M}_{2311} & 0 & \mathbb{M}_{2323} & \mathbb{M}_{2313} & 2 \mathbb{M}_{1322} \\
-\mathbb{M}_{1322} & \mathbb{M}_{1322} & 0 & -\mathbb{M}_{2313} & \mathbb{M}_{2323} & 2 \mathbb{M}_{2311} \\
-\mathbb{M}_{1222} & \mathbb{M}_{1222} & 0 & \mathbb{M}_{2213} & \mathbb{M}_{1123} & \mathbb{M}_{1111}-\mathbb{M}_{1122}
\end{array}\right]
$$

Tetragonal. Classes $4, \overline{4}$, and $4 / m$ :

$$
[\mathbb{M}] \equiv\left[\begin{array}{cccccc}
\mathbb{M}_{1111} & \mathbb{M}_{1122} & \mathbb{M}_{1133} & 0 & 0 & \mathbb{M}_{1112} \\
\mathbb{M}_{1122} & \mathbb{M}_{1111} & \mathbb{M}_{1133} & 0 & 0 & -\mathbb{M}_{1112} \\
\mathbb{M}_{3311} & \mathbb{M}_{3311} & \mathbb{M}_{3333} & 0 & 0 & 0 \\
0 & 0 & 0 & \mathbb{M}_{2323} & \mathbb{M}_{2313} & 0 \\
0 & 0 & 0 & -\mathbb{M}_{2313} & \mathbb{M}_{2323} & 0 \\
\mathbb{M}_{1211} & -\mathbb{M}_{1211} & 0 & 0 & 0 & \mathbb{M}_{1212}
\end{array}\right]
$$

Classes $4 m m m, 422,4 / m m$, and $\overline{4} 2 m$ :

$$
[\mathbb{M}] \equiv\left[\begin{array}{cccccc}
\mathbb{M}_{1111} & \mathbb{M}_{1122} & \mathbb{M}_{1133} & 0 & 0 & 0 \\
\mathbb{M}_{1122} & \mathbb{M}_{1111} & \mathbb{M}_{1133} & 0 & 0 & 0 \\
\mathbb{M}_{3311} & \mathbb{M}_{3311} & \mathbb{M}_{3333} & 0 & 0 & 0 \\
0 & 0 & 0 & \mathbb{M}_{2323} & & 0 \\
0 & 0 & 0 & 0 & \mathbb{M}_{2323} & 0 \\
0 & 0 & 0 & 0 & 0 & \mathbb{M}_{1212}
\end{array}\right] .
$$

Hexagonal. Classes $6, \overline{6}$, and $6 / m$ :

$$
[\mathbb{M}] \equiv\left[\begin{array}{cccccc}
\mathbb{M}_{1111} & \mathbb{M}_{1122} & \mathbb{M}_{1133} & 0 & 0 & 2 \mathbb{M}_{1222} \\
\mathbb{M}_{1122} & \mathbb{M}_{1111} & \mathbb{M}_{1133} & 0 & 0 & -2 \mathbb{M}_{1222} \\
\mathbb{M}_{3311} & \mathbb{M}_{3311} & \mathbb{M}_{3333} & 0 & 0 & 0 \\
0 & 0 & 0 & \mathbb{M}_{2323} & \mathbb{M}_{2313} & 0 \\
0 & 0 & 0 & -\mathbb{M}_{2313} & \mathbb{M}_{2323} & 0 \\
-2 \mathbb{M}_{122} & 2 \mathbb{M}_{1222} & 0 & 0 & 0 & \mathbb{M}_{1111}-\mathbb{M}_{1122}
\end{array}\right]
$$


Classes $\overline{6} m 2,622,6 m m$, and $6 / \mathrm{mmm}$ :

$$
[\mathrm{M}] \equiv\left[\begin{array}{cccccc}
\mathbb{M}_{1111} & \mathbb{M}_{1122} & \mathbb{M}_{1133} & 0 & 0 & 0 \\
\mathbb{M}_{1122} & \mathbb{M}_{1111} & \mathbb{M}_{1133} & 0 & 0 & 0 \\
\mathbb{M}_{3311} & \mathbb{M}_{3311} & \mathbb{M}_{3333} & 0 & 0 & 0 \\
0 & 0 & 0 & \mathbb{M}_{2323} & 0 & 0 \\
0 & 0 & 0 & 0 & \mathbb{M}_{2323} & 0 \\
0 & 0 & 0 & 0 & 0 & \mathbb{M}_{1111}-\mathbb{M}_{1122}
\end{array}\right] .
$$

Cubic. Classes 23 and $3 m$ :

$$
[\mathbb{M}] \equiv\left[\begin{array}{cccccc}
\mathbb{M}_{1111} & \mathbb{M}_{1122} & \mathbb{M}_{1133} & 0 & 0 & 0 \\
\mathbb{M}_{2211} & \mathbb{M}_{1111} & \mathbb{M}_{1122} & 0 & 0 & 0 \\
\mathbb{M}_{3311} & \mathbb{M}_{2211} & \mathbb{M}_{1111} & 0 & 0 & 0 \\
0 & 0 & 0 & \mathbb{M}_{1212} & 0 & 0 \\
0 & 0 & 0 & 0 & \mathbb{M}_{1212} & 0 \\
0 & 0 & 0 & 0 & 0 & \mathbb{M}_{1212}
\end{array}\right] .
$$

Classes $432, \overline{4} 3 m$, and $m 3 m$ :

$$
[\mathbb{M}] \equiv\left[\begin{array}{cccccc}
\mathbb{M}_{1111} & \mathbb{M}_{1122} & \mathbb{M}_{1122} & 0 & 0 & 0 \\
\mathbb{M}_{1122} & \mathbb{M}_{1111} & \mathbb{M}_{1122} & 0 & 0 & 0 \\
\mathbb{M}_{1122} & \mathbb{M}_{1122} & \mathbb{M}_{111} & 0 & 0 & 0 \\
0 & 0 & 0 & \mathbb{M}_{1212} & 0 & 0 \\
0 & 0 & 0 & 0 & \mathbb{M}_{1212} & 0 \\
0 & 0 & 0 & 0 & 0 & \mathbb{M}_{1212}
\end{array}\right]
$$

Isotropic.

$$
[\mathbb{M}] \equiv\left[\begin{array}{cccccc}
\mathbb{M}_{1111} & \mathbb{M}_{1122} & \mathbb{M}_{1122} & 0 & 0 & 0 \\
\mathbb{M}_{1122} & \mathbb{M}_{1111} & \mathbb{M}_{1122} & 0 & 0 & 0 \\
\mathbb{M}_{1122} & \mathbb{M}_{1122} & \mathbb{M}_{1111} & 0 & 0 & 0 \\
0 & 0 & 0 & \mathbb{M}_{1212} & 0 & 0 \\
0 & 0 & 0 & 0 & \mathbb{M}_{1212} & 0 \\
0 & 0 & 0 & 0 & 0 & \mathbb{M}_{1212}
\end{array}\right]
$$

with $2 \mathbb{M}_{1212}=\mathbb{M}_{1111}-\mathbb{M}_{1122}$.

We remark that, for isotropic materials and cubic crystals of classes $432, \overline{4} 3 \mathrm{~m}$, and $m 3 m$, the piezooptic tensor is symmetric, i.e., $\mathbb{M}_{i j h k}=\mathbb{M}_{h k i j}$ and accordingly the results obtained in [Mehrabadi and Cowin 1990] for the eigenvalues and eigentensor of the symmetric elasticity tensor still apply.

In particular we shall make use of the two following results from [Mehrabadi and Cowin 1990]. 
- Cubic crystals, classes $432, \overline{4} 3 m$, and $m 3 m$ :

$$
\begin{array}{r}
\mathbb{M}=\left(\mathbb{M}_{1111}+2 \mathbb{M}_{1122}\right) \frac{1}{3} \boldsymbol{I} \otimes \boldsymbol{I}+\left(\mathbb{M}_{1111}-\mathbb{M}_{1122}\right)\left(\sum_{k=1}^{3} \boldsymbol{E}_{k} \otimes \boldsymbol{E}_{k}-\frac{1}{3} \boldsymbol{I} \otimes \boldsymbol{I}\right) \\
+2 \mathbb{M}_{1212} \sum_{k=4}^{6} \boldsymbol{E}_{k} \otimes \boldsymbol{E}_{k},
\end{array}
$$

where

$$
\begin{aligned}
\boldsymbol{E}_{k} & =\frac{1}{\sqrt{2}} \boldsymbol{e}_{k} \otimes \boldsymbol{e}_{k}, \quad k=1,2,3, \\
\boldsymbol{E}_{4} & =\frac{1}{2}\left(\boldsymbol{e}_{2} \otimes \boldsymbol{e}_{3}+\boldsymbol{e}_{3} \otimes \boldsymbol{e}_{2}\right), \\
\boldsymbol{E}_{5} & =\frac{1}{2}\left(\boldsymbol{e}_{1} \otimes \boldsymbol{e}_{3}+\boldsymbol{e}_{3} \otimes \boldsymbol{e}_{1}\right), \\
\boldsymbol{E}_{6} & =\frac{1}{2}\left(\boldsymbol{e}_{2} \otimes \boldsymbol{e}_{1}+\boldsymbol{e}_{1} \otimes \boldsymbol{e}_{2}\right) .
\end{aligned}
$$

- Isotropic materials:

$$
\mathbb{M}=\left(\mathbb{M}_{1111}+2 \mathbb{M}_{1122}\right) \frac{1}{3} \boldsymbol{I} \otimes \boldsymbol{I}+\left(\mathbb{M}_{1111}-\mathbb{M}_{1122}\right) \mathbb{D},
$$

where $\mathbb{D}: \mathrm{Sym} \rightarrow$ Dev is given by

$$
\mathbb{D}=\sum_{k=1}^{6} \boldsymbol{E}_{k} \otimes \boldsymbol{E}_{k}-\frac{1}{3} \boldsymbol{I} \otimes \boldsymbol{I},
$$

i.e., $\mathbb{D}[\boldsymbol{T}]=\operatorname{dev} \boldsymbol{T}$.

\section{Acknowledgments}

The research leading to these results is within the scope of CERN R\&D Experiment 18 "Crystal Clear Collaboration" and the PANDA Collaboration at GSI-Darmstadt. It has received funding from the Progetto Strategico di Ateneo 2017: "Scintillating crystals: an interdisciplinary, applications-oriented approach aimed to the scientific knowledge and process control for application concerning life-quality improvement".

\section{References}

[Aben and Guillemet 1993] H. Aben and C. Guillemet, Photoelasticity of glass, Springer, 1993.

[Authier 2003] A. Authier (editor), International tables for crystallography, vol. D: Physical properties of crystals, Springer, 2003.

[Bain 2019] A. K. Bain, Crystal optics: properties and applications, Wiley, Weinheim, Germany, 2019.

[Born and Wolf 1999] M. Born and E. Wolf, Principles of optics: electromagnetic theory of propagation, interference and diffraction of light, 7th ed., Cambridge University, 1999. 
[Brewster 1830] D. Brewster, Papers on optics, Royal Society of London, 1830.

[Davì 2015] F. Davì, "On the Bertin surfaces of photoelastic crystals", J. Opt. Soc. Am. A 32:12 (2015), 2323-2337.

[Kuske and Robertson 1974] A. Kuske and C. Robertson, Photoelastic stress analysis, Wiley, London, 1974.

[Mehrabadi and Cowin 1990] M. M. Mehrabadi and S. C. Cowin, "Eigentensors of linear anisotropic elastic materials", Quart. J. Mech. Appl. Math. 43:1 (1990), 15-41.

[Montalto et al. 2019] L. Montalto, P. P. Natali, L. Scalise, N. Paone, F. Davì, D. Rinaldi, G. Barucca, and P. Mengucci, "Quality control and structural assessment of anisotropic scintillating crystals", Crystals 9:7 (2019), art. id. 376.

[Nye 1985] J. F. Nye, Physical properties of crystals: their representation by tensors and matrices, Oxford University, 1985.

[Perelomova and Tagieva 1983] N. V. Perelomova and M. M. Tagieva, Problems in crystal physics: with solutions, Mir, Moscow, 1983.

[Rinaldi et al. 2018] D. Rinaldi, F. Davì, and L. Montalto, "On the photoelastic constants and the Brewster law for stressed tetragonal crystals”, Math. Methods Appl. Sci. 41:8 (2018), 3103-3116.

[Sirotin and Shaskolskaya 1982] Y. I. Sirotin and M. P. Shaskolskaya, Fundamentals of crystal physics, Mir, Moscow, 1982.

[Sokolnikoff 1956] I. S. Sokolnikoff, Mathematical theory of elasticity, 2nd ed., McGraw-Hill, New York, 1956.

Received 30 Jul 2019. Revised 4 Aug 2019. Accepted 18 Jan 2020.

FABRIZIO DAVÌ: davi@univpm.it

Dipartimento di Ingegneria Civile, Edile e Architettura, Centro Interdipartimentale di Analisi

Globale dei Cristalli, Università Politecnica delle Marche, Ancona, Italy 
EDITORIAL BOARD

ANTONIO CARCATERRA

ERIC A. CARLEN

FRANCESCO DELL'ISOLA

RAFFAELE ESPOSITO

ALBERT FANNJIANG

Gilles A. FranCFORT

PiERANGELO MARCATI

JEAN-JACQUES MARIGO

PETER A. MARKOWICH

MARTIN OSTOJA-STARZEWSKI

PIERRE SEPPECHER

DAVID J. STEIGMANN

PAUl STEINMANN

PierRe M. SuQueT

MANAGING EDITORS

MICOL AMAR

EMILIO BARCHIESI

ANGELA MADEO

MARTIN OSTOJA-STARZEWSKI

ADVISORY BOARD

ADNAN AKAY

Holm AltenbaCH

MICOL AMAR

HARM ASKES

TEODOR ATANACKOVIĆ

VICTOR BERDICHEVSKY

GUY BOUCHITTÉ

ANDREA BRAIDES

ROBERTO CAMASSA

MAURO CARFORE

ERIC DARVE

FELIX DARVE

ANNA DE MASI

Gianpietro Del Piero

EMMANUELE Di BENEDETTO

VICTOR A. EREMEYEV

BERNOLD FIEDLER

IRENE M. GAMBA

DAVID Y. GAO

Sergey GaVRILyuk

Timothy J. HEALEY

DOMINIQUe JEULiN

ROGER E. KHAYAT

Corrado LATTANZIO

ROBERT P. LIPTON

ANGELO LUONGO

ANGELA MADEO

JUAN J. MANFREDI

CARLO MARCHIORO ANIL MisRa

ROBERTO NATALINI PATRIZIO NEFF

Thomas J. PENCE

ANDREY PIATNITSKI

ERRICO PRESUTTI

MARIO Pulvirenti

LUCIO RUSSO

Miguel A. F. SAnjuaN

PATRICK SElVAdurai

MiroslaV ŠILHAVÝ

GUIDO SWEERS

ANTOINETTE TORDESILLAS

LEV TRUSKINOVSKY

JUAN J. L. VELÁZQUEZ

VINCENZO VESPRI

ANGELO VULPIANI msp.org/memocs

Università di Roma "La Sapienza", Italia

Rutgers University, USA

(CO-CHAIR) Università di Roma "La Sapienza", Italia

(TREASURER) Università dell'Aquila, Italia

University of California at Davis, USA

(CO-CHAIR) Université Paris-Nord, France

Università dell' Aquila, Italy

École Polytechnique, France

DAMTP Cambridge, UK, and University of Vienna, Austria

(CHAIR MANAGING EDITOR) Univ. of Illinois at Urbana-Champaign, USA

Université du Sud Toulon-Var, France

University of California at Berkeley, USA

Universität Erlangen-Nürnberg, Germany

LMA CNRS Marseille, France

Università di Roma "La Sapienza”, Italia

Università degli Studi dell'Aquila, Italy

Université de Lyon-INSA (Institut National des Sciences Appliquées), France

(CHAIR MANAGING EDITOR) Univ. of Illinois at Urbana-Champaign, USA

Carnegie Mellon University, USA, and Bilkent University, Turkey

Otto-von-Guericke-Universität Magdeburg, Germany

Università di Roma "La Sapienza", Italia

University of Sheffield, UK

University of Novi Sad, Serbia

Wayne State University, USA

Université du Sud Toulon-Var, France

Università di Roma Tor Vergata, Italia

University of North Carolina at Chapel Hill, USA

Università di Pavia, Italia

Stanford University, USA

Institut Polytechnique de Grenoble, France

Università dell'Aquila, Italia

Università di Ferrara and International Research Center MEMOCS, Italia

Vanderbilt University, USA

Gdansk University of Technology, Poland

Freie Universität Berlin, Germany

University of Texas at Austin, USA

Federation University and Australian National University, Australia

Université Aix-Marseille, France

Cornell University, USA

École des Mines, France

University of Western Ontario, Canada

Università dell' Aquila, Italy

Louisiana State University, USA

Università dell'Aquila, Italia

Université de Lyon-INSA (Institut National des Sciences Appliquées), France

University of Pittsburgh, USA

Università di Roma "La Sapienza", Italia

University of Kansas, USA

Istituto per le Applicazioni del Calcolo "M. Picone", Italy

Universität Duisburg-Essen, Germany

Michigan State University, USA

Narvik University College, Norway, Russia

Università di Roma Tor Vergata, Italy

Università di Roma "La Sapienza", Italia

Università di Roma "Tor Vergata", Italia

Universidad Rey Juan Carlos, Madrid, Spain

McGill University, Canada

Academy of Sciences of the Czech Republic

Universität zu Köln, Germany

University of Melbourne, Australia

École Polytechnique, France

Bonn University, Germany

Università di Firenze, Italia

Università di Roma La Sapienza, Italia

MEMOCS (ISSN 2325-3444 electronic, 2326-7186 printed) is a journal of the International Research Center for the Mathematics and Mechanics of Complex Systems at the Università dell'Aquila, Italy.

Cover image: "Tangle” by @ John Horigan; produced using the Context Free program (contextfreeart.org).

\section{PUBLISHED BY}

\section{I. mathematical sciences publishers} nonprofit scientific publishing http://msp.org/ 
Mathematics and Mechanics of Complex Systems vol. 8 no. 1

Second-order work criterion and divergence criterion: a full 1 equivalence for kinematically constrained systems

Jean Lerbet, Noël Challamel, François Nicot and Felix

Darve

On the generalization of the Brewster Law

Fabrizio Davì

IBVP for electromagneto-elastic materials: variational approach

Amirhossein Amiri-Hezaveh, Pouyan Karimi and

Martin Ostoja-Starzewski

Equilibrium theory for a lipid bilayer with a conforming cytoskeletal membrane

Brett Hendrickson, Milad Shirani and David J.

Steigmann

MEMOCS is a journal of the International Research Center for the Mathematics and Mechanics of Complex Systems at the Università dell' Aquila, Italy.

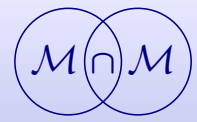

